\title{
Exact solutions and dynamic properties of perturbed nonlinear schrödinger equation arised from nano optical fibers
}

\author{
Shuxin Bao \\ School of mathematical science \\ Daqing Normal University \\ Daqing, 163712 Heilongjiang, China \\ Email*: a18546992666@163.com
}

September 23, 2021

\begin{abstract}
The main idea of this paper is to investigate the exact solutions and dynamic properties in optical nanofibers, which is modeled by space-time fractional perturbed nonlinear schrödinger equation involving Kerr law nonlinearity with conformal fractional derivative. Firstly, by the complex fractional traveling wave transformation, the traveling wave system of the original equation is obtained, then a conserved quantity, namely the Hamiltonian is constructed, and the qualitative analysis of this system is conducted via this quantity by classifying the equilibrium points. Moreover, the prior estimate of the existence of the soliton and periodic solution is established via the bifurcation method. Furthermore, all exact traveling wave solutions are constructed to illustrate our results explicitly by the complete discrimination system for polynomial method.

Keywords: perturbed nonlinear schrödinger equation; conformal fractional derivative; prior estimate; bifurcation method; complete discrimination system for polynomial method.
\end{abstract}

\section{Introduction}

For hundred of years, partial differential equation plays a vital role in many fields of science, and constructing exact solution to it could help us gain a deep insight into the phenomena revealed. However, traditional integer-order equation sometimes could not meet the requirement of modeling some special problems such as anomalous diffusion [1], thus the fractional calculus is proposed to handle this. Fractional calculus has many definitions, for example, the traditional Riemann-Liouville (RL) definition [2], modified RL definition [3] and conformal definition [4]. However, the classical RL definition is very complex 
to apply, and the modified RL definition has already been proved wrong [5-6], thus choosing a proper fractional definition is an important and difficult task.

In this paper, we consider the following space-time fractional perturbed nonlinear schrödinger equation

$$
i q_{t}^{\alpha}+a q_{x}^{2 \alpha}+b|q|^{2} q-i\left(\sigma q_{x}^{\alpha}-\lambda\left(|q|^{2} q\right)_{x}^{\alpha}-\gamma\left|q^{2}\right|_{x}^{\alpha} q\right)=0,
$$

with conformal fractional derivative, where $\alpha$ is the corresponding fractional order, $q(x, t)$ is the complex valued function defining wave profile in optical fibers, $a$ and $b$ represent the group velocity dispersion and nonlinear term, respectively, $\sigma$ is the intermodal dispersion, $\lambda$ is the self-steepeninng perturbation term and $\gamma$ is the nonlinear dispersion coefficient. This equation has a broad applications in modeling light wave in nano optical fibers. Especially, when the external electric field exists, this equation can be used to solve non-harmonic motion of electrons bound in molecules [7], so constructing exact solutions to this equation is of great significance. In [8], some optical solitons and singular periodic wave solutions are constructed by the extended Sinh-Gordon equation expansion method, and $\mathrm{W}$-shaped solitons are shown by Al-Ghafri and his colleagues [9]. In this paper, we conduct qualitative and quantitative analysis to (1) by the complete discrimination system for polynomial method (CDSPM). The topological structure of this equation is shown and the prior estimate of the existence of soliton and periodic solution is also presented. Moreover, to verify our conclusion explicitly, all exact traveling wave solutions, namely the classification of traveling wave solutions is gotten. All conditions of parameters are discussed, thus this paper contains all results in the existing literatures, and some new solutions are obtained. The CDSPM is proposed by Liu [10-12], and has successfully applied to a series of integer-order [13-14] and fractional order equations [15-19], then Kai found that this method could also be used to conduct qualitative analysis [20], and via the bifurcation method, we can even establish the existence of the soliton and periodic solution [21].

The construction of this paper is as follows. The corresponding traveling wave system is given in Sec. 2 and qualitative analysis is conducted. Moreover, the existence of the soliton and periodic solution is also established in this section. In Sec. 2, all single traveling wave solutions are constructed to verify our conclusion explicitly, and concrete examples under concrete parameters are also shown to ensure the existence of each solution. In the final, a brief discussion is given.

\section{Dynamic properties of Eq. (1)}

By setting

$$
q(x, t)=u(\xi) e^{i(\phi(\xi)-w t)},
$$

where $\xi=\frac{x^{\alpha}}{\alpha}-\frac{v t^{\alpha}}{\alpha}[18],(1)$ becomes

$$
-(v+\sigma) u^{\prime}+2 a u^{\prime} \phi^{\prime}+a u \phi^{\prime \prime}+(3 \lambda+2 \gamma) u^{2} u^{\prime}=0,
$$


for the real part, and

$$
a u^{\prime \prime}+w u+b u^{3}+(v+\sigma) u \phi^{\prime}-a u\left(\phi^{\prime}\right)^{2}-\lambda u^{3} \phi^{\prime}=0,
$$

for the imaginary part. Substituting (3) into (4) yields

$$
u^{\prime \prime}=A_{3} u^{5}+A_{2} u^{3}+A_{1} u+A_{0} u^{-3},
$$

where $A_{1}=\frac{C\left(\lambda+2 \gamma-2 a w+(v+\sigma)^{2}\right)}{2 a^{2}}, A_{2}=\frac{\lambda(v+\sigma)-2 a b}{2 a^{2}}, A_{3}=\frac{4 \lambda \gamma+4 \gamma^{2}-3 \lambda^{2}}{16 a^{2}}, A_{0}=$ $\frac{C^{2}}{a^{2}}$ and $C$ is a constant of integration. By setting $u^{2}=V$, we have

$$
V^{\prime \prime}=\frac{8 A_{3}}{3} V^{3}+3 A_{2} V^{2}+4 A_{1} V+4 A_{0}
$$

Multiplying (6) with $V^{\prime}$ and integrating it once, we have

$$
\left(V^{\prime}\right)^{2}=a_{4} V^{4}+a_{3} V^{3}+a_{2} V^{2}+a_{1} V+a_{0},
$$

where $a_{4}=\frac{2 A_{3}}{3}, a_{3}=A_{2}, a_{2}=2 A_{1}, a_{1}=4 A_{0}$ and $a_{0}$ is a constant of integration.

(6) is equivalent to the following dynamic system

$$
\begin{aligned}
V^{\prime} & =U, \\
U^{\prime} & =\frac{8 A_{3}}{3} V^{3}+3 A_{2} V^{2}+4 A_{1} V+4 A_{0},
\end{aligned}
$$

and thus the corresponding Hamiltonian is given by

$$
H(U, V)=U^{2}-\left(a_{4} V^{4}+a_{3} V^{3}+a_{2} V^{2}+a_{1} V\right) .
$$

Now let us show that the Hamiltonian (9) is a conserved quantity. Taking derivative of right side of (9) with respect to $\xi$, we have

$2 U U^{\prime}-\left(4 a_{4} V^{3}+3 a_{3} V^{2}+2 a_{2} V+a_{1}\right) V^{\prime}=2 U\left[U^{\prime}-\left(\frac{8 A_{3}}{3} V^{3}+3 A_{2} V^{2}+4 A_{1} V+4 A_{0}\right)\right]=0$,

which just proved our conclusion, and we can also conclude that the global phase portraits to system (8) is just the contour lines of the Hamiltonian (9). In the following, we shall conduct qualitative analysis through this quantity by introducing the complete discrimination system.

From the Hamiltonian (9), we can see that the derivative of the potential energy is given by [20]

$$
U_{1}^{\prime}(V)=-4 a_{4}\left(V^{3}+b_{2} V^{2}+b_{1} V+b_{0}\right),
$$

where $b_{2}=\frac{3 a_{3}}{4 a_{4}}, b_{1}=\frac{a_{2}}{2 a_{4}}$ and $b_{0}=\frac{a_{1}}{4 a_{4}}$. By introducing following discrimination system

$$
\begin{aligned}
& \Delta=-27\left(\frac{2}{27} b_{2}^{3}+b_{0}-\frac{b_{1} b_{2}}{3}\right)^{2}-4\left(b_{1}-\frac{b_{2}^{2}}{3}\right)^{3}, \\
& D=b_{1}-\frac{1}{3} b_{2}^{2} .
\end{aligned}
$$


we can see that four cases need to be discussed here.

Case 1. $D<0, \Delta=0$, we can get

$$
U^{\prime}(V)=-4 a_{4}(V-s)^{2}(V-l) .(s \neq l)
$$

$(s, 0)$ is a cuspidal point and $(l, 0)$ is a center for $a_{4}>0$ and a saddle point when $a_{4}<0$. For example, when $a_{3}=\frac{4}{3}, a_{2}=a_{1}=0$, we have $s=0, l=1$, and corresponding global phase portraits are shown in Fig. 1 when $a_{4}= \pm 1$.

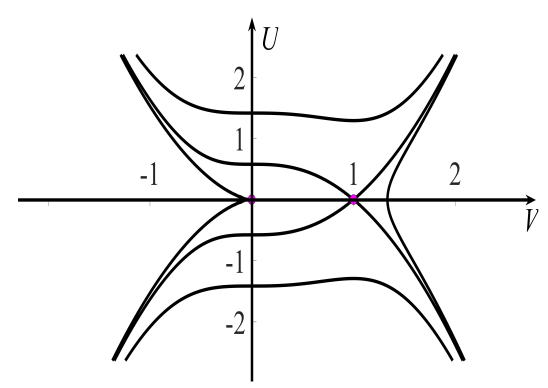

(a)

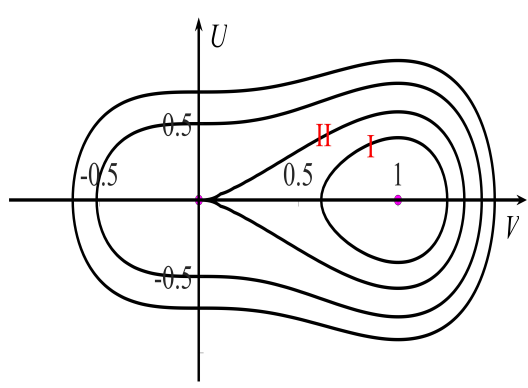

(b)

Figure 1: The phase portraits of (8) in Case 1: (a) $a_{4}=1$, (b) $a_{4}=-1$.

From Fig. 1b, we can see that trajectory $\mathrm{I}$ is a closed orbit with a center inside, which just indicate the existence of the periodic solution, and trajectory II is a homoclinic orbit, which indicate the existence of the bell-shaped soliton solution [21].

Case 2. $D=0, \Delta=0$, we have

$$
U^{\prime}(V)=-4 a_{4}(V-s)^{3}
$$

We can only get one equilibrium point $(s, 0)$ here. It is a cuspidal point when $a_{4}>0$ and a center when $a_{4}<0$. For example, when $a_{3}=a_{2}=a_{1}=0$, we have $s=0$, and the corresponding global phase portraits can be seen in Fig. 2 when $a_{4}= \pm 1$. From Fig. $2 \mathrm{~b}$, we can also conclude that the original equation has periodic solution.

Case 3. $\Delta<0$, we have

$$
U_{1}^{\prime}(V)=(V-s)\left(V^{2}+s V+l\right) \cdot\left(s^{2}-4 l<0\right)
$$

There is only one equilibrium point $(s, 0)$ in this case. It is a saddle point when $a_{4}>0$ and a center when $a_{4}<0$. So this case is very similar to Case 2. For example, when $a_{3}=a_{1}=0$ and $a_{2}=2$, we have $s=-1$, and the global phase portrait is given in Fig. 3 when $a_{4}= \pm 1$.

Case 4. $D<0, \Delta>0$, we have

$$
U_{1}^{\prime}(V)=-4 a_{4}(V-s)(V-l)(V-m) .
$$




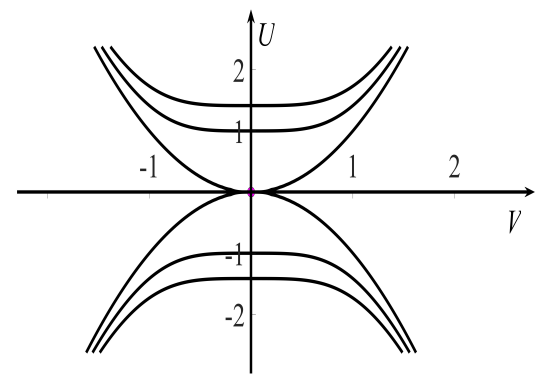

(a)

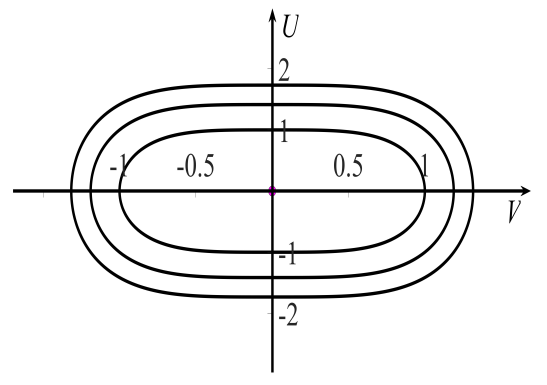

(b)

Figure 2: The phase portraits of (8) in Case 2: (a) $a_{4}=1$, (b) $a_{4}=-1$

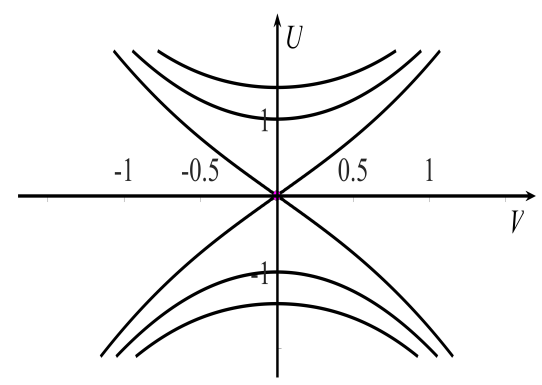

(a)

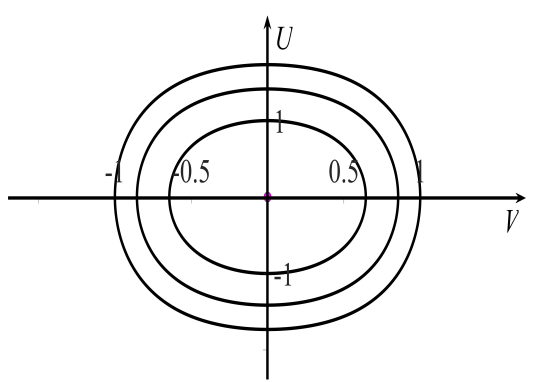

(b)

Figure 3: The phase portraits of (8) in Case 3: (a) $a_{4}=1$, (b) $a_{4}=-1$ 
This case is rather interesting due to that there are three equilibrium points $(s, 0),(l, 0)$ and $(m, 0)$ here. When $a_{4}>0,(s, 0)$ and $(m, 0)$ are two saddle points and $(l, 0)$ is a center, and whereas for $a_{4}<0,(s, 0)$ and $(m, 0)$ are two centers and $(l, 0)$ is a saddle point. Concrete examples of global phase portraits when $s=-m=1, l=0$ and $a_{4}= \pm 1$ are given in Fig. 4 .

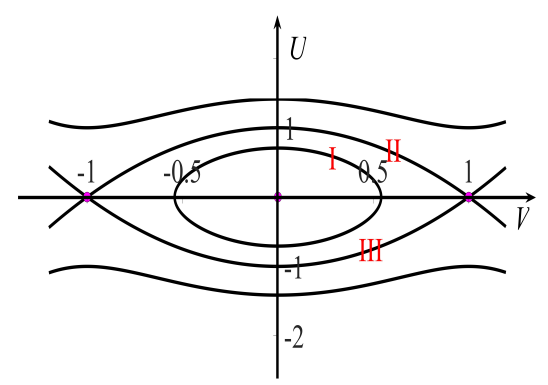

(a)

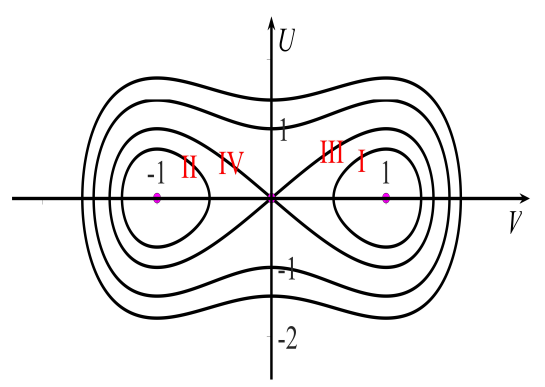

(b)

Figure 4: The phase portraits of (8) in Case 4: (a) $a_{4}=1$, (b) $a_{4}=-1$

For Fig. 4a, we can see that trajectory I is a closed orbit with a center inside, which indicates the existence of the periodic solution, and trajectory II and III are two heteroclinic orbits, which indicates the existence of the kink and anti-kink solitary wave solution, respectively. Fig. 4b is a "figure eight loop" with trajectoris I and II are two closed orbits with a center inside, which indicate the existence of the periodic solution, and trajectories III and IV are two homoclinic orbits, which means the corresponding equation has bright and dark bell-shaped soliton solution.

Now we have showed the topological structure of the system (8) and established the existence of the soliton and the periodic solution. In order to verify the conclusion explicitly, we construct the classification of traveling wave solutions to (7) by the CDSPM.

\subsection{Traveling wave solutions to (7)}

In this section, we construct all traveling wave solutions, namely the classification of traveling wave solutions to (7). We only focus on the condition of $a_{4}>0$, and $a_{4}<0$ could also be treated similarly.

By taking the following transformation

$$
\varphi=\left(a_{4}\right)^{1 / 4}\left(u+\frac{a_{3}}{4 a_{4}}\right), \xi_{1}=\left(a_{4}\right)^{1 / 4} \xi,
$$

(7) becomes

$$
\left(\varphi_{\xi_{1}}^{\prime}\right)^{2}=\varphi^{4}+p \varphi^{2}+q \varphi+r=F(\varphi),
$$


where $p=\frac{a_{2}}{\sqrt{a_{4}}}, q=\left(\frac{a_{3}^{3}}{8 a_{2}^{4}}-\frac{a_{2} a_{3}}{2 a_{4}}+a_{1}\right) a^{-1 / 4}$ and $r=\frac{-a_{3}^{4}}{256 a_{4}^{3}}+\frac{a_{2} a_{3}^{2}}{16 a_{4}^{2}}-\frac{a_{1} a_{3}}{4 a_{4}}+a_{0}$. First, we need to introducing the following complete discrimination system

$$
\begin{aligned}
& D_{1}=4, D_{2}=-p, D_{3}=-2 p^{3}+8 p r-9 q^{2} \\
& D_{4}=-p^{2} q^{2}+4 p^{4} q+36 p q^{2} r-32 p^{2} q^{2}-\frac{27}{4} q^{4}+64 r^{3}, E_{2}=9 p^{2}-32 p r .
\end{aligned}
$$

The complete discrimination system (12) given in Sec. 2 is the third order form, and here is fourth order. By discussing the relation between the parameters and the coefficients, we shall see that every condition of parameters are considered, thus what we have gotten is the classification of traveling wave solutions.

Case $1 D_{2}<0, D_{3}=0$ and $D_{4}=0, F(\varphi)$ has a pair of double conjugate complex roots, namely

$$
F(\varphi)=\left(\varphi^{2}+s^{2}\right)^{2},(s>0)
$$

by substituting (20) into (7), we have the following solution

$$
\varphi=s \tan \left(s\left(\xi_{1}-\xi_{0}\right)\right)
$$

where $\xi_{0}$ is a constant of integration. (21) is a trigonometric function periodic solution. For example if $p=4, q=0$ and $r=4$, then we have $s=2$ and the solution (21) is given by

$$
\varphi=2 \tan 2\left(\xi_{1}-\xi_{0}\right) .
$$

Case 2 When $D_{2}=D_{3}=D_{4}=0 . F(\varphi)$ has a real root zero of multiplicities four, namely

$$
F(\varphi)=\varphi^{4}
$$

which leads to

$$
\xi_{1}-\xi_{0}=\int \frac{d \varphi}{\varphi^{2}}=-\frac{1}{\varphi^{-1}} .
$$

For example, when $p=q=r=0$, we have

$$
\varphi=-\frac{1}{\xi_{1}-\xi_{0}} \text {. }
$$

Case 3 When $D_{2}>0, D_{3}=D_{4}=0$ and $E_{2}>0, F(\varphi)$ has two double distinct real roots, then we have

$$
F(\varphi)=(\varphi-s)^{2}(\varphi-l)^{2},(s>l)
$$

which yields

$$
\pm\left(\xi_{1}-\xi_{0}\right)=\int \frac{d \varphi}{(\varphi-\mu)(\varphi-\nu)}=\frac{1}{\mu-\nu} \ln \left|\frac{\varphi-\mu}{\varphi-\nu}\right| .
$$

If $\varphi>s$ or $\varphi<l$, we can get

$$
\varphi=\frac{l-s}{2}\left[\operatorname{coth} \frac{ \pm(s-l)\left(\xi_{1}-\xi_{0}\right)}{2}-1\right]+s,
$$


when $l<\varphi<s$, we have

$$
\varphi=\frac{l-s}{2}\left[\tanh \frac{ \pm(s-l)\left(\xi_{1}-\xi_{0}\right)}{2}-1\right]+l .
$$

(29) is a solitary wave solution. (21) and (29) have verified the conclusion given in Sec. 2 that when $a_{4}>0,(7)$ has periodic and soliton solution. This show that the qualitative results obtained are truly correct.

For example, when $p=-8, r=0$, and $q=116$, we have $s=-l=2$, then solitary wave solution (29) is given by

$$
\varphi=-2\left[\tanh \frac{ \pm 2\left(\xi_{1}-\xi_{0}\right)}{2}-1\right]-2 .
$$

Case $4 D_{2}>0, D_{3}=D_{4}=E_{2}=0 . \quad F(\varphi)=(\varphi-s)^{3}(\varphi-l)$, then the solution is given by

$$
\pm\left(\xi_{1}-\xi_{0}\right)=\int \frac{d \varphi}{(\varphi-s) \sqrt{(\varphi-s)(\varphi-l)}}=\frac{2}{l-s} \sqrt{\frac{\varphi-s}{\varphi-l}},
$$

thus the solution in explicit form is

$$
\varphi=\frac{4(s-l)}{(l-s)^{2}\left(\xi_{1}-\xi_{0}\right)^{2}-4}+s,
$$

which is a rational solution. For example, when $p=-6, q=8$ and $r=-3$, we have $l=1, s=-3$, then

$$
\varphi=\frac{4}{1-4\left(\xi_{1}-\xi_{0}\right)^{2}}-3
$$

Case $5 D_{2} D_{3}<0$, and $D_{4}=0$, we have

$$
F(\varphi)=(\varphi-l)^{2}\left[(\varphi+l)^{2}+s^{2}\right]
$$

and then we can get

$$
\pm\left(\xi_{1}-\xi_{0}\right)=\frac{1}{\sqrt{4 l^{2}+s^{2}}} \ln \left|\frac{\mu \varphi+\delta-\sqrt{(\varphi+l)^{2}+s^{2}}}{\varphi-l}\right|,
$$

where

$$
\mu=\frac{3 l}{\sqrt{4 l^{2}+s^{2}}}
$$

and

$$
\delta=\sqrt{4 l^{2}+s^{2}}-\frac{3 l^{2}}{\sqrt{4 l^{2}+s^{2}}} .
$$

Thus

$$
\varphi=\frac{e^{ \pm \sqrt{4 l^{2}+s^{2}}\left(\xi_{1}-\xi_{0}\right)}-\mu+\sqrt{4 l^{2}+s^{2}}(2-\mu)}{\left(e^{ \pm \sqrt{4 l^{2}+s^{2}}\left(\xi_{1}-\xi_{0}\right)}-\mu\right)^{2}-1} .
$$


For example, when $p=-3, q=-2 \sqrt{2}$, and $r=6$, we have $l=\sqrt{2}, s=1$, then the solution is given by

$$
\varphi=\frac{e^{ \pm 3\left(\xi_{1}-\xi_{0}\right)}-4 \sqrt{2}+6}{\left(e^{ \pm 3\left(\xi_{1}-\xi_{0}\right)}-\sqrt{2}\right)^{2}-1} .
$$

Case $6 D_{i}>0(i=2,3,4) . F(\varphi)$ is given by

$$
H(\varphi)=\left(\varphi-\alpha_{1}\right)\left(\varphi-\alpha_{2}\right)\left(\varphi-\alpha_{3}\right)\left(\varphi-\alpha_{4}\right),
$$

where $\alpha_{1}>\alpha_{2}>\alpha_{3}>\alpha_{4}$. When $\alpha_{4}>0$, we have

$$
\begin{aligned}
& \varphi=\frac{\alpha_{2}\left(\alpha_{1}-\alpha_{4}\right) \operatorname{sn}^{2}\left(\frac{\sqrt{\left(\alpha_{1}-\alpha_{3}\right)\left(\alpha_{2}-\alpha_{4}\right)}}{2}\left(\xi_{1}-\xi_{0}\right), m\right)-\alpha_{1}\left(\alpha_{2}-\alpha_{4}\right)}{\left(\alpha_{1}-\alpha_{4}\right) \operatorname{sn}^{2}\left(\frac{\sqrt{\left(\alpha_{1}-\alpha_{3}\right)\left(\alpha_{2}-\alpha_{4}\right)}}{2}\left(\xi_{1}-\xi_{0}\right), m\right)-\left(\alpha_{2}-\alpha_{4}\right)} \\
& \left(\varphi>\alpha_{1} \text { or } \varphi<\alpha_{4}\right) \\
& \omega=\frac{\alpha_{4}\left(\alpha_{2}-\alpha_{3}\right) \operatorname{sn}^{2}\left(\frac{\sqrt{\left(\alpha_{1}-\alpha_{3}\right)\left(\alpha_{2}-\alpha_{4}\right)}}{2}\left(\xi_{1}-\xi_{0}\right), m\right)-\alpha_{3}\left(\alpha_{2}-\alpha_{4}\right)}{\left(\alpha_{2}-\alpha_{3}\right) \operatorname{sn}^{2}\left(\frac{\sqrt{\left(\alpha_{1}-\alpha_{3}\right)\left(\alpha_{2}-\alpha_{4}\right)}}{2}\left(\xi_{1}-\xi_{0}\right), m\right)-\left(\alpha_{2}-\alpha_{4}\right)}, \\
& \left(\alpha_{3}<\varphi<\alpha_{2}\right)
\end{aligned}
$$

where $m^{2}=\frac{\left(\alpha_{1}-\alpha_{4}\right)\left(\alpha_{2}-\alpha_{3}\right)}{\left(\alpha_{1}-\alpha_{3}\right)\left(\alpha_{2}-\alpha_{4}\right)}$.

For $a_{4}<0$, similarly we can get

$$
\begin{aligned}
& \varphi=\frac{\alpha_{3}\left(\alpha_{1}-\alpha_{2}\right) \operatorname{sn}^{2}\left(\frac{\sqrt{\left(\alpha_{1}-\alpha_{3}\right)\left(\alpha_{2}-\alpha_{4}\right)}}{2}\left(\xi_{1}-\xi_{0}\right), m\right)-\alpha_{2}\left(\alpha_{1}-\alpha_{3}\right)}{\left(\alpha_{1}-\alpha_{2}\right) \operatorname{sn}^{2}\left(\frac{\sqrt{\left(\alpha_{1}-\alpha_{3}\right)\left(\alpha_{2}-\alpha_{4}\right)}}{2}\left(\xi_{1}-\xi_{0}\right), m\right)-\left(\alpha_{1}-\alpha_{3}\right)}, \\
& \left(\alpha_{1}>\varphi>\alpha_{2}\right),
\end{aligned}
$$

and

$$
\begin{aligned}
& \varphi=\frac{\alpha_{1}\left(\alpha_{3}-\alpha_{4}\right) \operatorname{sn}^{2}\left(\frac{\sqrt{\left(\alpha_{1}-\alpha_{3}\right)\left(\alpha_{2}-\alpha_{4}\right)}}{2}\left(\xi_{1}-\xi_{0}\right), m\right)-\alpha_{4}\left(\alpha_{3}-\alpha_{1}\right)}{\left(\alpha_{3}-\alpha_{4}\right) \operatorname{sn}^{2}\left(\frac{\sqrt{\left(\alpha_{1}-\alpha_{3}\right)\left(\alpha_{2}-\alpha_{4}\right)}}{2}\left(\xi_{1}-\xi_{0}\right), m\right)-\left(\alpha_{3}-\alpha_{1}\right)}, \\
& \left(\alpha_{4}<\varphi<\alpha_{3}\right)
\end{aligned}
$$

where $m^{2}=\frac{\left(\alpha_{1}-\alpha_{2}\right)\left(\alpha_{3}-\alpha_{4}\right)}{\left(\alpha_{1}-\alpha_{3}\right)\left(\alpha_{2}-\alpha_{4}\right)}$. Solutions (41-44) are all elliptic function double periodic solutions. For instance, when $p=-5, q=0, r=4$ and $\varphi>2$, we have $\alpha_{1}=2, \alpha_{2}=1, \alpha_{3}=-1, \alpha_{4}=-2$, and thus the solution is given by

$$
\varphi=\frac{4 \operatorname{sn}^{2}\left(\frac{\sqrt{9}}{2}\left(\xi_{1}-\xi_{0}\right), \frac{8}{9}\right)-6}{4 \operatorname{sn}^{2}\left(\frac{\sqrt{9}}{2}\left(\xi_{1}-\xi_{0}\right), \frac{8}{9}\right)-3} .
$$

Case $7 D_{2} D_{3} \geq 0$ and $D_{4}<0 . F(\varphi)$ is given by

$$
F(\varphi)=(\varphi-\mu)(\varphi-\nu)\left((\varphi-l)^{2}+s^{2}\right)
$$


where $\mu>\nu$ and $s>0$. By setting

$$
\begin{gathered}
a=\frac{1}{2}(\mu+\nu) c-\frac{1}{2}(\mu-\nu) d, \\
b=\frac{1}{2}(\mu+\nu) d-\frac{1}{2}(\mu-\nu) c \\
c=\mu-l-\frac{s}{m_{1}} \\
d=\mu-l-s m_{1} \\
E=\frac{s^{2}+(\mu-l)(\nu-l)}{s(\mu-\nu)} \\
m_{1}=E+\sqrt{E^{2}+1}
\end{gathered}
$$

we can get

$$
\varphi=\frac{a \operatorname{cn}\left(\frac{\sqrt{\mp 2 s m_{1}(\mu-\nu)}}{2 m m_{1}}\left(\xi_{1}-\xi_{0}\right), m\right)+b}{c \operatorname{cn}\left(\frac{\sqrt{\mp 2 s m_{1}(\mu-\nu)}}{2 m m_{1}}\left(\xi_{1}-\xi_{0}\right), m\right)+d},
$$

where $m^{2}=\frac{1}{1+m_{1}^{2}}$. For instance, when $p=3, q=-4$, then $\mu=1, \nu=-1$ and $l=0, s=2$, thus the following solution could just be obtained

$$
\varphi=-\operatorname{cn}\left(\frac{\sqrt{\mp 2 s m_{1}(\mu-\nu)}}{2 m m_{1}}\left(\xi_{1}-\xi_{0}\right), m\right),
$$

which is also an elliptic function double periodic solution.

Case $8 D_{2} D_{3} \leq 0$ and $D_{4}>0$, we can get

$$
F(\varphi)=\left(\left(\varphi-l_{1}\right)^{2}+s_{1}^{2}\right)\left(\left(\varphi-l_{2}\right)^{2}+s_{2}^{2}\right),\left(s_{1} \geq s_{2}>0\right)
$$

By setting

$$
\begin{gathered}
a=l_{1} c+s_{1} d, \\
b=l_{1} d-s_{1} c, \\
c=-s_{1}-\frac{s_{2}}{m_{1}}, \\
d=l_{1}-l_{2}, \\
E=\frac{\left(l_{1}-l_{2}\right)^{2}+s_{1}^{2}+s_{2}^{2}}{2 s_{1} s_{2}}, \\
m_{1}=E+\sqrt{E^{2}-1},
\end{gathered}
$$

we have

$$
\varphi=\frac{a \operatorname{sn}\left(\eta\left(\xi_{1}-\xi_{0}\right), m\right)+b \operatorname{cn}\left(\eta\left(\xi_{1}-\xi_{0}\right), m\right)}{c \operatorname{sn}\left(\eta\left(\xi_{1}-\xi_{0}\right), m\right)+d \operatorname{cn}\left(\eta\left(\xi_{1}-\xi_{0}\right), m\right)}
$$


where $m^{2}=\frac{m_{1}^{2}-1}{m_{1}^{2}}$ and $\eta=\frac{s_{2} \sqrt{\left(c^{2}+d^{2}\right)\left(m_{1}^{2} c^{2}+d^{2}\right)}}{c^{2}+d^{2}}$. For example, when $p=5$, $q=4$, we have $l_{1}=l_{2}=0, s_{1}=1, s_{2}=2$, then

$$
\varphi=\frac{\operatorname{cn}\left(4\left(\xi_{1}-\xi_{0}\right), \frac{3}{4}\right)}{\operatorname{sn}\left(4\left(\xi_{1}-\xi_{0}\right), \frac{3}{4}\right)} .
$$

Case $9 D_{2}, D_{3}>0$, and $D_{4}=0 . F(\varphi)$ is given by

$$
F(\varphi)=(\varphi-s)(\varphi-l)(\varphi-m)^{2} \cdot(s>l)
$$

By setting

$$
c=\frac{\alpha_{1}-\alpha_{2}}{2}\left(\frac{\alpha_{1}+\alpha_{2}}{2}-\alpha_{3}\right)
$$

we have

$$
\pm\left(\xi_{1}-\xi_{0}\right)=\int \frac{d \varphi}{\left(\varphi-\alpha_{3}\right) \sqrt{\left.\varphi-\alpha_{1}\right)\left(\varphi-\alpha_{2}\right)}},
$$

whose solution is given by

$$
\left(\xi_{1}-\xi_{0}\right)=-\frac{1}{\sqrt{c^{2}-1}} \ln \left|\frac{y-c_{1}}{y+c_{1}}\right|,\left(c^{2}-1>0\right)
$$

and

$$
\left(\xi_{1}-\xi_{0}\right)=-\sqrt{\left(1-c^{2}\right)} \arctan \frac{c+1}{1-c} y, \quad\left(c^{2}-1<0\right)
$$

where $c_{1}=\sqrt{\frac{c+1}{c-1}}$ and $y=\sqrt{1-\frac{\alpha_{1}-\alpha_{2}}{\left(\varphi-\frac{\alpha_{1}+\alpha_{2}}{2}\right)+\frac{\alpha_{1}-\alpha_{2}}{2}}}$, which is a triangular function periodic solution. For example, when $p=-1, q=0$, we have $\alpha_{1}=1, \alpha_{2}=$ -1 , and $\alpha_{3}=0$, which leads to

$$
\varphi=\frac{1}{\cosh 2\left(\xi_{1}-\xi_{0}\right)},
$$

which is also a soliton solution.

\section{Conclusion}

In this paper, we consider a space-time fractional perturbed nonlinear schrödinger equation arised from nano optical fibers. By taking the complex fractional traveling wave transformation, the traveling wave system of the original equation is gotten, then the corresponding Hamiltonian is constructed, and the qualitative analysis is conduct by introducing the complete discrimination system. The topological structure is given and the existence of the soliton and periodic solution is established via the bifurcation method. To verify our conclusion explicitly, every kind of traveling wave solutions is constructed by the CDSPM, and some of them are new.

\section{Conflict of Interest}

The authors declare that they have no conflict of interest.

Data Availability Statements

All data generated or analyzed during this study are included in this article. 


\section{References}

[1] Zheng B, Kai Y, Xu W, et al. Exact traveling and non-traveling wave solutions of the time fractional reaction-diffusion equation. Physica A: Statistical Mechanics and its Applications, 2019, 532: 121780.

[2] Lakshmikantham V, Vatsala A S. Theory of fractional differential equations. Nonlinear Analysis, 2008, 69(8): 2677-2682.

[3] Jumarie G. Modified Riemann-Liouville derivative and fractional Taylor series of nondifferentiable functions further results. Computers and Mathematics With Applications, 2006, 51(9): 1367-1376.

[4] Khalil R, Al Horani M, Yousef A, et al. A new definition of fractional derivative. Journal of computational and applied mathematics, 2014, 264: 65-70.

[5] Cheng-shi Liu. Counterexamples on Jumarie's two basic fractional calculus formulae. Communications in Nonlinear science and Numerical Simulation, 2015, 22(1-3): 92-94.

[6] Cheng-shi Liu. Counterexamples on Jumarie's three basic fractional calculus formulae for non-differentiable continuous functions. Chaos Solitons and Fractals, 2018, 109: 219-222.

[7] Biswas A, Triki H, Zhou Q, et al. CubicCquartic optical solitons in Kerr and power law media[J]. Optik, 2017, 144: 357-362.

[8] Sulaiman T A, Bulut H, Baskonus H M. Optical solitons to the fractional perturbed NLSE in nano-fibers. Discrete and Continuous Dynamical Systems-S, 2020, 13(3): 925 .

[9] Al-Ghafri K S, Krishnan E V, Biswas A. W-shaped and other solitons in optical nanofibers. Results in Physics, 2021, 23: 103973.

[10] Cheng-Shi L. Classification of all single travelling wave solutions to CalogeroCDegasperisCFocas equation. Communications in Theoretical Physics, 2007, 48(4): 601.

[11] Cheng-Shi L. All single traveling wave solutions to $(3+1)$-dimensional NizhnokCNovikovCVeselov equation. Communications in theoretical physics, 2006, 45(6): 991-992.

[12] Cheng-shi Liu. The classification of travelling wave solutions and superposition of multi-solutions to Camassa-Holm equation with dispersion. Chinese Physics, 2007, 16(7): 1832.

[13] Kai Y. The classification of the single travelling wave solutions to the variant Boussinesq equations. Pramana, 2016, 87(4): 1-5. 
[14] Kai Y, Zheng B, Yang N, et al. Exact single traveling wave solutions to generalized $(2+1)$-dimensional Gardner equation with variable coefficients. Results in Physics, 2019, 15: 102527.

[15] Cao D. The classification of the single traveling wave solutions to the timefraction Gardner equation. Chinese Journal of Physics, 2019, 59: 379-392.

[16] Guan B, Li S, Chen S, et al. The classification of single traveling wave solutions to coupled time-fractional KdV-Drinfel'd-Sokolov-Wilson system. Results in Physics, 2019, 13: 102291.

[17] Chen S, Li Y, Li Y, et al. Variant wave propagation patterns by coupled Bossinesq equations. Results in Physics, 2021, 24: 104147.

[18] Chen S, Li Y, Jiang M, et al. Abundant traveling wave solutions to an intrinsic fractional discrete nonlinear electrical transmission line. Results in Physics, 2021, 28: 104587.

[19] Xiao X, Yin Z. Exact single travelling wave solutions to the fractional perturbed GerdjikovCIvanov equation in nonlinear optics. Modern Physics Letters B, 2021: 2150377.

[20] Kai Y, Chen S, Zheng B, et al. Qualitative and quantitative analysis of nonlinear dynamics by the complete discrimination system for polynomial method. Chaos, Solitons and Fractals, 2020, 141: 110314.

[21] Kai Y, Chen S, Zhang K, et al. A study of the shallow water waves with some Boussinesq-type equations. Waves in Random and Complex Media, 2021: 1-18. 